\title{
Effects of surface roughness on local friction and temperature distributions in a steel-on-steel fretting contact
}

\author{
W. Qin ${ }^{\text {a, }}{ }^{*}$, X. Jin a, A. Kirk a, P. H. Shipway a, W. Sun ${ }^{\text {a }}$ \\ *qinwj@bit.edu.cn \\ ${ }^{a}$ Faculty of Engineering, University of Nottingham, Nottingham NG7 2RD, UK \\ ${ }^{\mathrm{b}}$ School of Mechanical Engineering, Beijing Institute of Technology, Beijing 10081, China
}

\begin{abstract}
It has generally been assumed that initial surface roughness has significant influences on local frictional shear stresses and flash temperatures in fretting contacts. However, since these hypotheses are difficult to directly measure, the frictional shear stress and temperature rise distributions in a steelon-steel fretting contact are investigated by finite element method. The rough contacting surfaces were modelled as fractal surfaces by the Weierstrass-Mandelbrot function. The simulation results show that the frictional shear stress distribution resulted from the rough contact model is discrete, and the local stresses are highly concentrated which result in higher peak temperature rise than that from the smooth contact model. The influences of the plasticity of materials, load and frequency on the temperature rise are also discussed.
\end{abstract}

\section{KEYWORDS}

\section{Surface roughness; friction; temperature; fretting contact}

\section{Introduction}

Fretting is a wear process which occurs in loaded contacts between two bodies when they are subjected to small oscillating displacements relative to each other. During this process, frictional power is dissipated over the relatively small area of true contact and thus causes high flash temperatures in these areas and a relatively steep temperature gradient into the substrate. The role of temperature in fretting has generally been attributed to changes associated with oxidation process which are thought to exert a significant influence on the rate and mechanisms of wear [1-5]. As such, friction and friction-induced thermal histories in fretting contacts have been the subject of investigation by many researchers.

Some research has focussed on investigation of friction behaviour at fretting contacts [6-9]; a number of researchers have investigated the temperature profile in the contact resulting from the dissipation of frictional power [2,10-16]. Since direct temperature measurement close to the fretting surface itself is often impractical, the actual contact temperature can only be estimated using analytical models and computer simulation tools and thus efficient models are needed at the design stage to predict the friction-induced temperature rise under such conditions [1, 15]. For example, Wen and Khonsari presented an analytical approach for obtaining the transient temperature profile for different oscillating heat sources on a semi-infinite body and the analytic expressions for maximum surface temperature for these heat sources were provided based on an extensive number of simulations [15]. Jin et al. adopted a mathematical model and developed a finite element (FE) model to predict the temperature rise in a fretting contact based upon the frictional power dissipation, and explored the 
influences of various factors such as the presence of an oxide debris layer and frequency on the contact temperature within a stainless steel contact $[2,16]$.

It has been found that surface roughness has significant influence on friction, wear and temperature rise in contacts [17-26]. Some researchers have studied the effects of surface roughness on friction by experiments [17-21]. For example, $\mathrm{Lu}$ et al. experimentally examined friction torque under different surface roughness and different texture directions with a torsional fretting wear device with a flat-onflat contact and found that friction torque and accumulated dissipated energy at first increase and then decrease as the surface roughness increases [17]. Others adopted numerical methods to analyse the effects of surface roughness on friction and contact temperature [22-26]. For example, Reichert et al. determined the influence of elastic and plastic deformation on the friction coefficient as the effect of surface flattening in the run-in phase was taken into account with a numerical approach based on the FE model in which surface roughness of turning processes and plastic hardening due to deformation of the asperities are considered [22]. In some cases where experiments are difficult to carry out, numerical calculations can often provide an alternative to give some indication about the possible effects of certain parameters. In the case of the contact temperature which is difficult to measure experimentally, Attia et al. have developed analytical models to predict the disturbed temperature field around the contact asperity in fretting contacts by assuming that the asperity has a uniform square cross section $[14,25,26]$. They presented the dimension of the thermally disturbed zone and the effect of reciprocating motion on the maximum surface temperature rise in dimensionless form.

Although analytical models can provide some general conclusions about the temperature field in fretting contacts, it is difficult to predict the frictional and thermal behaviour of a real fretting tribosystem of complex geometry with realistic surface roughness; however, more significant progress in this regard can be achieved with numerical methods, e.g., finite element (FE) modelling. In this paper, the dynamic local contact frictional shear stress and temperature rise distributions in a steel-on-steel contact during the fretting process are studied by the FE method taking into account the initial surface roughness. The rough contacting surfaces are modelled as fractal surfaces by the WeierstrassMandelbrot (W-M) function, and the FE models (including the surface roughness) are developed for the cylinder-on-flat configuration used in the experimental work (see Fig. 1) which results in a line contact on a macroscopic scale. FE analyses were carried out and the effects of surface roughness on the distributions of frictional shear stress and temperature rise are investigated by comparing the results obtained with those from the smooth contact model.

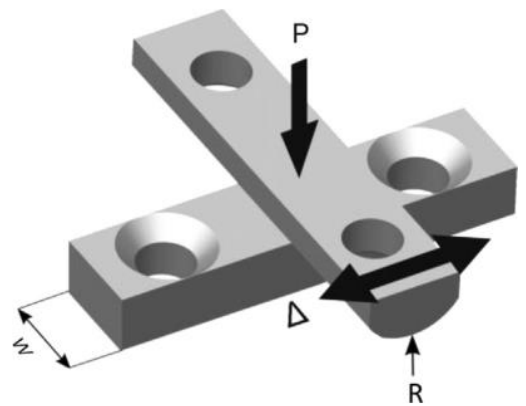

Fig. 1 Cylinder-on-flat configuration for fretting test. 


\section{Representation of the rough surfaces}

\subsection{Description of surface roughness by fractal geometry}

Statistical surface parameters which are often used to describe surface roughness are related to the sample size [27]; in light of this, surface topography in more recent contact analyses is commonly described by fractal geometry since this is characterized by the properties of continuity, nondifferentiability, scale invariance, and self-affinity [28-32]. In this study, the surface roughness is described by fractal geometry and its two-dimensional (2-D) surface profile height is given by the W-M function [33]

$$
z(x)=G^{D-1} \sum_{n=n_{1}}^{n_{2}} \frac{\cos 2 \pi \gamma^{n} x}{\gamma^{(2-D) n}}, \quad 1<D<2
$$

where $D(1<D<2)$ is the fractal dimension which determines the relative contributions of highand low-frequency components in the surface profile, $G$ is the fractal scale coefficient which reflects the amplitudes of the surface profile, $\gamma(\gamma>1)$ is a constant which controls the density of frequencies in the surface profile ( $\gamma=1.5$ is typical for most surfaces), $n$ is the fractal scale index and $n_{1}$ and $n_{2}$ are the lowest and highest cut-off indexes of frequency. Letting $\omega=\gamma^{n}$ be the spatial frequency of the profile, then $\omega_{L}$ is the starting frequency determined by the sample length $L$ as $\omega_{L}=1 / L$, and $\omega_{U}$ is the upper limit of frequency which is determined by the profile resolution $\delta$ as $\omega_{U}=1 / 2 \delta$.

The fractal parameter $D$ is determined by the structure function method [34] in which the surface roughness is modelled as a series data with fractal characteristics which satisfy

$$
S(\tau)=<[z(x+\tau)-z(x)]^{2}>=C \tau^{4-2 D}
$$

in which $<[z(x+\tau)-z(x)]^{2}>$ is the average of square deviation in height of points pairs separated by an interval along the profile, $C$ is a constant and $\tau$ is the arbitrary interval.

According to a number of various interval $\tau$, the corresponding values of $S(\tau)$ can be calculated using Eq. (2). Drawing the $\log -\log$ plot $\log S-\log \tau$ as shown in Fig. 2, the fractal dimension can be determined by the slop of the diagram line $k$ as

$$
D=2-k / 2
$$

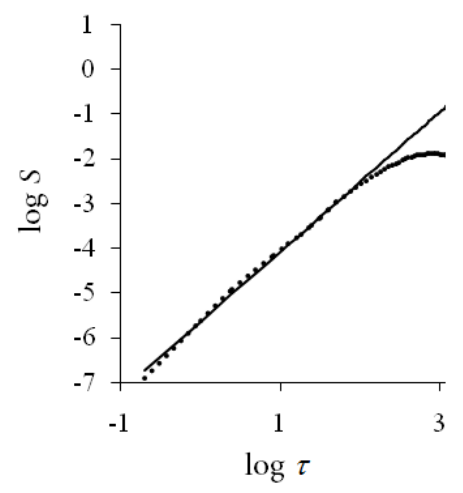

Fig. $2 \log S-\log \tau$ plot to derive the slop of the diagram line. 
According to $\mathrm{E}\left[z^{2}(x)\right]=R_{q}{ }^{2}$ in which $R_{q}$ is the root mean square roughness of surface, the fractal roughness can be determined as [34]

$$
G^{D-1}=2 R_{q}\left(\omega_{L} \omega_{U}\right)^{2-D}\left(\frac{2-D}{\omega_{U}^{4-2 D}-\omega_{L}^{4-2 D}}\right)
$$

\subsection{Representation of the rough surfaces of the specimens}

In this study, the coordinates of $4 \mathrm{~mm}$ length of the surface profile (made up of 8000 data points for each specimen) are obtained via non-contact laser measurement using Mitaka PF-60, which uses a laser autofocus method to determine the co-ordinates of the profile with a resolution of $0.1 \mu \mathrm{m}$ in height direction and $0.5 \mu \mathrm{m}$ in lateral direction. The $1 \mathrm{~mm}$ length of the measurement data of the cylinder $(R=6 \mathrm{~mm})$ and flat specimens $(W=10 \mathrm{~mm})$ are shown in Fig. 3.

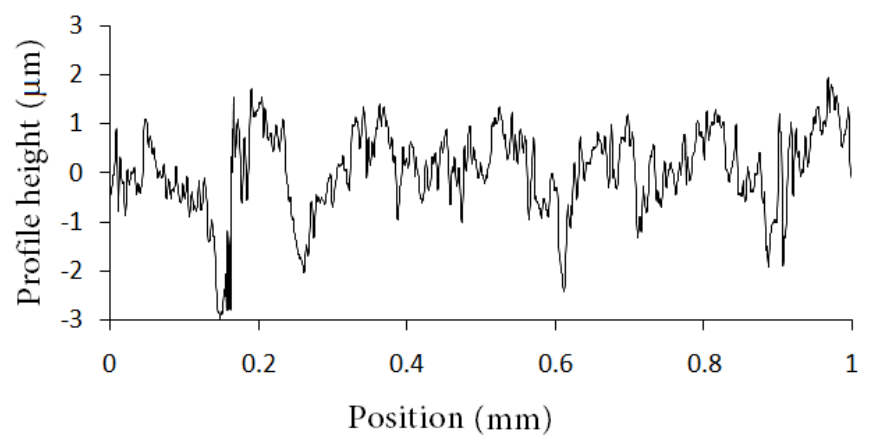

(a)

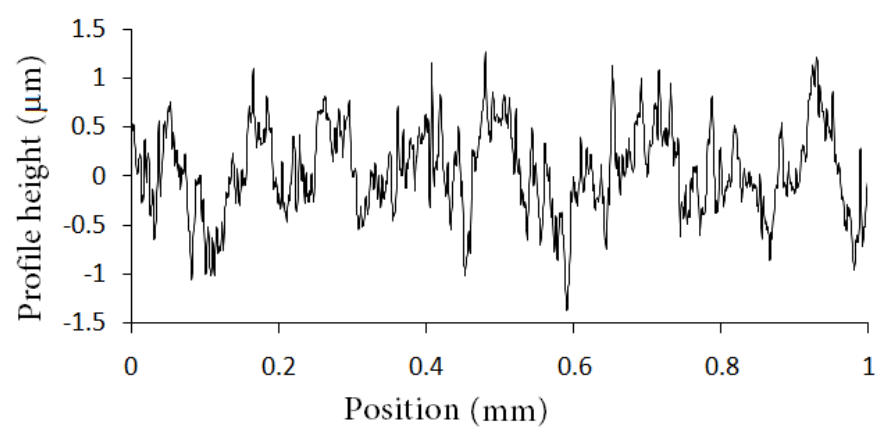

(b)

Fig. 3 Measured profile heights: (a) cylinder surface; (b) flat surface.

In order to implement a flexible and scale-independent rough surface profile in the FE model, the W-M function (Eq. (1)) is used to characterize the surface profile using the method described in Section 2.1. As the fretting amplitude $(\Delta)$ is $50 \mu \mathrm{m}$ and the contact width is less than $100 \mu \mathrm{m}$, the width of the rough contact surfaces to be modelled is limited to $300 \mu \mathrm{m}$. The fractal dimensions and fractal scale coefficients of the fractal surface profiles of the cylinder sample and the flat sample shown in Table 1 are generated using the measured profile height data. The modelled $300 \mu \mathrm{m}$ long profile heights for the cylinder and the flat samples compared with the measurement data are shown in Fig. 4. 
Table 1 Fractal dimensions and fractal scale coefficients

\begin{tabular}{ccc}
\hline & Fractal dimension $D$ & Fractal scale coefficient $G$ \\
\hline Cylinder & 1.4108 & 5.72 \\
Flat & 1.3586 & 4.35 \\
\hline
\end{tabular}

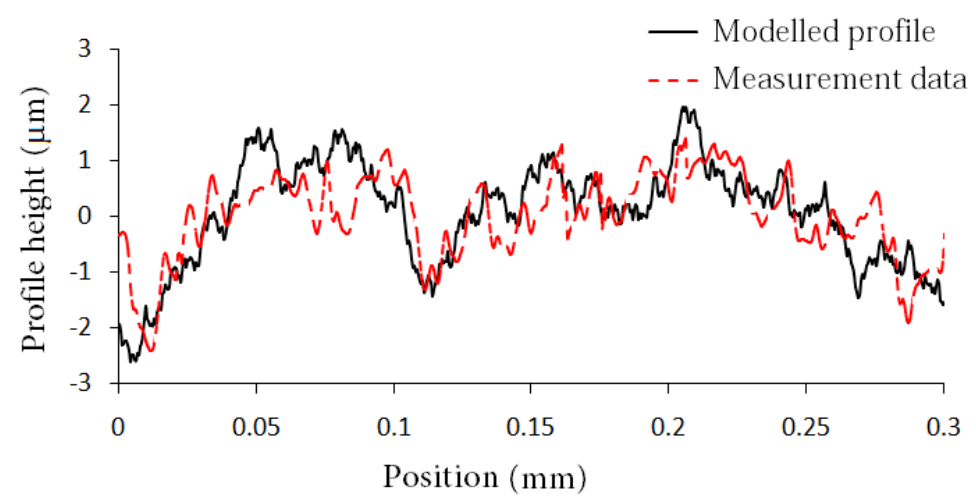

(a)

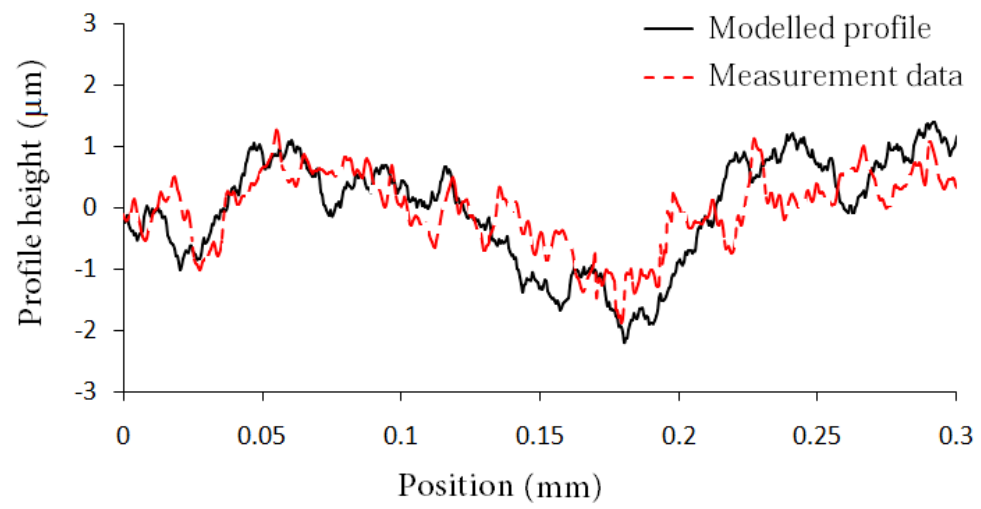

(b)

Fig. 4 Modelled $300 \mu \mathrm{m}$ long profile heights and measurement data:

(a) cylinder surface; (b) flat surface.

\section{Coupled temperature-displacement analysis for the cylinder-on-flat fretting contact}

\subsection{Properties of material}

The material of the cylinder and the flat used in this study is an alloy steel, S132. The mechanical and thermal properties of this material which are adopted in this paper are presented in Table 2 .

Table 2 Mechanical and thermal properties of S132 [35, 36].

\begin{tabular}{ccccc}
\hline $\begin{array}{c}\text { Young's modulus } E \\
(\mathrm{GPa})\end{array}$ & $\begin{array}{c}\text { Poisson's } \\
\text { ratio } v\end{array}$ & $\begin{array}{c}\text { Density } \rho \\
\left(\mathrm{kgm}^{-3}\right)\end{array}$ & $\begin{array}{c}\text { Conductivity } k \\
\left(\mathrm{Wm}^{-1} \mathrm{~K}^{-1}\right)\end{array}$ & $\begin{array}{c}\text { Specific heat } C \\
\left(\mathrm{~J} \mathrm{~kg}^{-1} \mathrm{~K}^{-1}\right)\end{array}$ \\
\hline 206.8 & 0.28 & 7850 & 39 & 520 \\
\hline
\end{tabular}


Due to stress concentration associated with the rough surfaces, plastic deformation is likely to occur in the contacting asperities. Therefore, the plastic properties of the material are also included in the FE model. The normalised stress-strain curve for this alloy (from the literature) is shown in Fig. 5.

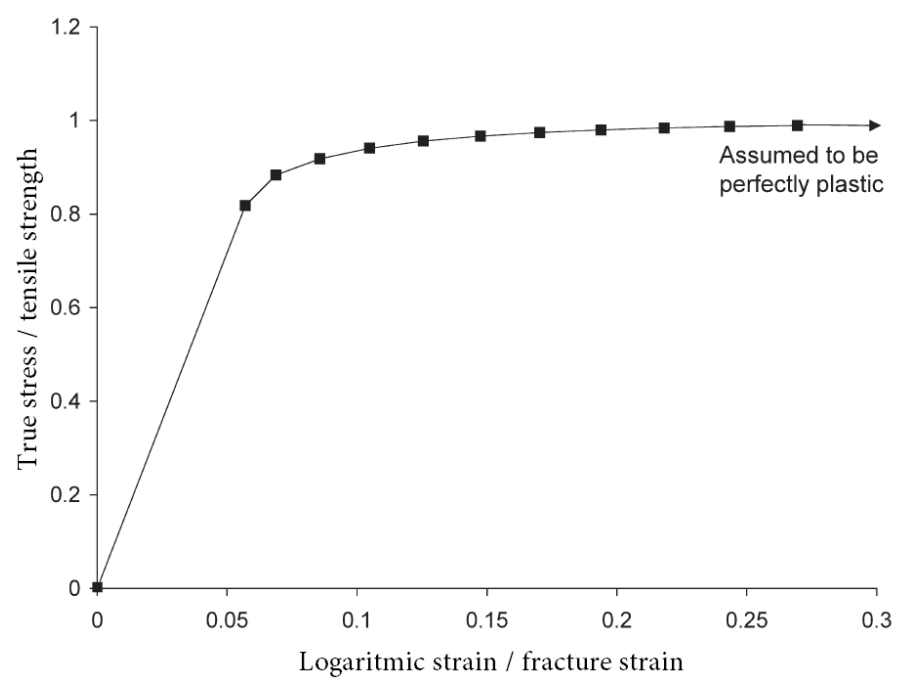

Fig. 5 Normalised stress-strain curve for S132 [35].

\subsection{Development of the FE model}

The basic cylinder-on-flat configuration was composed of a semi-circular body with $6 \mathrm{~mm}$ radius and a flat with $10 \mathrm{~mm}$ width; the FE models (including both smooth contact surfaces and rough contact surfaces) have been developed to simulate the fretting contact and the resulting temperature rise using a nonlinear ABAQUS code. The contact is simplified as a 2-D problem, and thus 2-D coupled temperature-displacement plane strain elements are employed. In the contact zone, an extremely fine mesh was generated as shown in Fig. 6. For the smooth contact models, a $10 \mu \mathrm{m}$ element size was used; for the rough contact models, $2 \mu \mathrm{m}, 1 \mu \mathrm{m}$ and $0.5 \mu \mathrm{m}$ elements were used to investigate which size of element is required to adequately capture the contact behaviour.

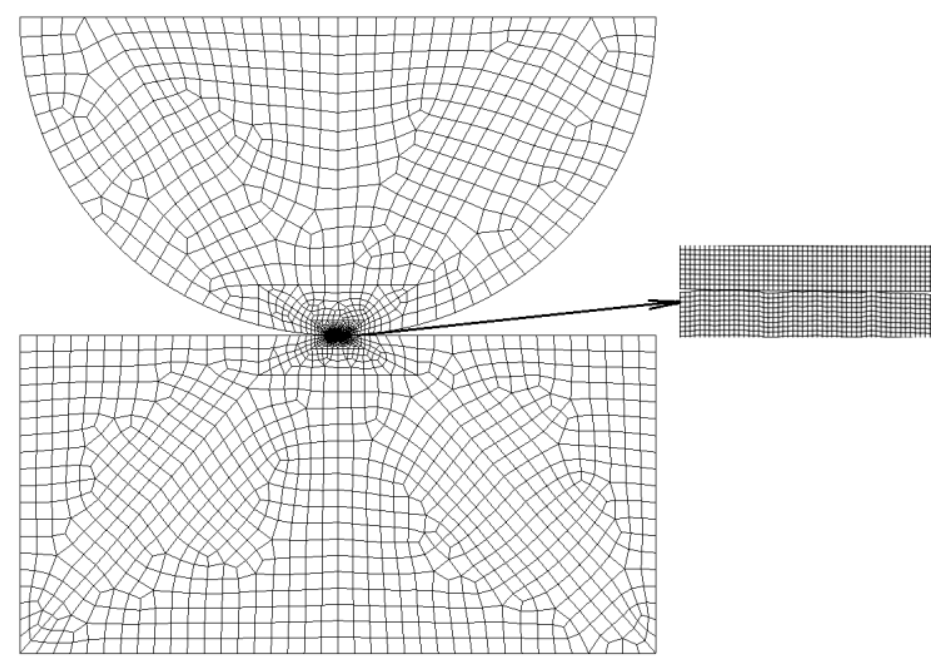

Fig. 6 Wireframe image of the finite element model. 
Fixed boundary conditions were applied to the nodes along the base of the flat specimen, and a frictional contact was applied between surfaces of the cylinder and the flat for which a Coulomb friction model was adopted to calculate the frictional stresses due to sliding in a fretting contact. A sinusoidal horizontal displacement was applied to the nodes along the top surface of the cylinder specimen and a vertical concentrated force was applied to the centre of this surface.

\subsection{Coupled temperature-displacement analysis}

In this study, the coupled temperature-displacement analysis was carried out to obtain the stress and temperature responses. The coupled equations illustrated in matrix representation are [37]

$$
\left[\begin{array}{ll}
K_{u u} & K_{u \theta} \\
K_{\theta u} & K_{\theta \theta}
\end{array}\right]\left\{\begin{array}{l}
\Delta u \\
\Delta \theta
\end{array}\right\}=\left\{\begin{array}{l}
R_{u} \\
R_{\theta}
\end{array}\right\}
$$

where $\Delta u$ and $\Delta \theta$ are the respective corrections to the incremental displacement and temperature, $K_{u u}$, $K_{u \theta}, K_{\theta u}$ and $K_{\theta \theta}$ are the stiffness submatrices, and $R_{u}$ and $R_{\theta}$ are the mechanical and thermal residual vectors respectively.

In the thermal analysis, conduction is considered to be the major term of heat dissipation in the fretting process, and thus the heat loss due to convection and radiation from the surfaces is neglected [2]. As only the heat converted by frictional work is investigated in this study, the additional temperature rise resulting from plastic energy dissipation is not considered here. The heat flux density, $q_{g}$, generated by the interface element due to frictional heat generation is given by [37]

$$
q_{g}=\eta \tau_{f} \dot{\gamma}
$$

where $\tau_{f}$ is the frictional shear stress, $\dot{\gamma}$ is the slip rate, and $\eta$ determines the fraction of the frictional work converted to heat that enters the contacting bodies. Under dry sliding conditions, it can be assumed that all of the energy dissipated through friction is transferred to the contacting bodies as heat [38]. As the heat source only affects a small area, the temperatures for both bodies are expected to be similar. Therefore, the thermal energy is assumed to partition equally into the two bodies [2]; that is to say, $\eta=0.5$. Moreover, heat flux on the non-contacting surfaces is assumed to be zero.

Using the models with smooth contact and rough contact developed in Section 3.2, the steady-state coupled temperature-displacement simulation was implemented using ABAQUS software under the conditions shown in Table 3 according to the test procedure presented in Ref. 39.

Table 3 Simulation conditions

\begin{tabular}{cccc}
\hline $\begin{array}{c}\text { Applied normal } \\
\text { load }(\mathrm{N})\end{array}$ & $\begin{array}{c}\text { Displacement } \\
\text { amplitude }(\mu \mathrm{m})\end{array}$ & $\begin{array}{c}\text { Coefficient of } \\
\text { friction }\end{array}$ & $\begin{array}{c}\text { Frequency } \\
(\mathrm{Hz})\end{array}$ \\
\hline 250 & 50 & 0.8 & 200 \\
\hline
\end{tabular}

\section{Simulation results}

\subsection{Determination of element size for rough contact models}

Fig. 7 (a) and Fig. 7 (b) give the frictional shear stress and temperature rise distributions near the contact area along the surface of the flat specimen in the tenth cycle from the rough contact models with $2 \mu \mathrm{m}, 1 \mu \mathrm{m}$ and $0.5 \mu \mathrm{m}$ element sizes in the contact zone. For the sake of comparison, the frictional shear stress and temperature rise distribution results from the rough contact model with elastic material (i.e. without plasticity) are shown in Fig. 8 (a) and Fig. 8 (b). It can be seen that the peak values of the frictional shear stresses resulted from the elastic model with different element sizes 
in the contact zone are quite different and the greatest frictional shear stress from the model with a 0.5 $\mu \mathrm{m}$ element size is much higher than that from the model with a $2 \mu \mathrm{m}$ element size. But the peak values of the frictional shear stresses resulting from the elastic-plastic model with different element sizes in the contact zone are very similar because of the surface flattening when the material plasticity is included. Moreover, the temperature rise distributions resulting from both the elastic and the elasticplastic models with different element sizes in the contact zone are very similar (the largest difference is less than $0.5 \mathrm{~K}$ ). Therefore, for the sake of computational efficiency, the rough contact model with a $2 \mu \mathrm{m}$ element size in the contact zone is adopted in the simulations presented throughout this paper.

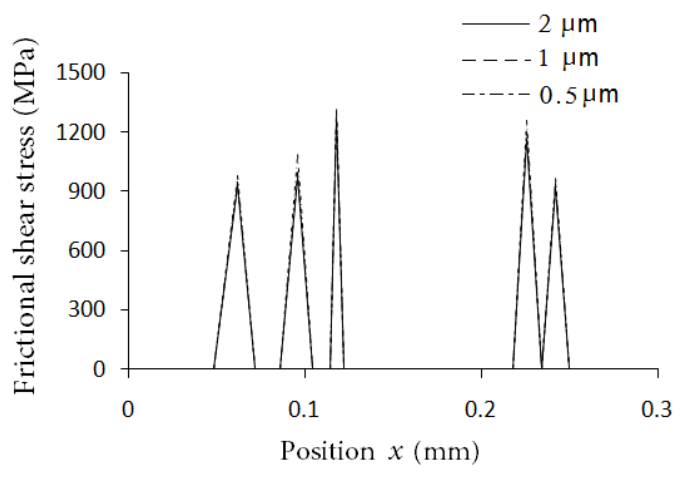

(a)

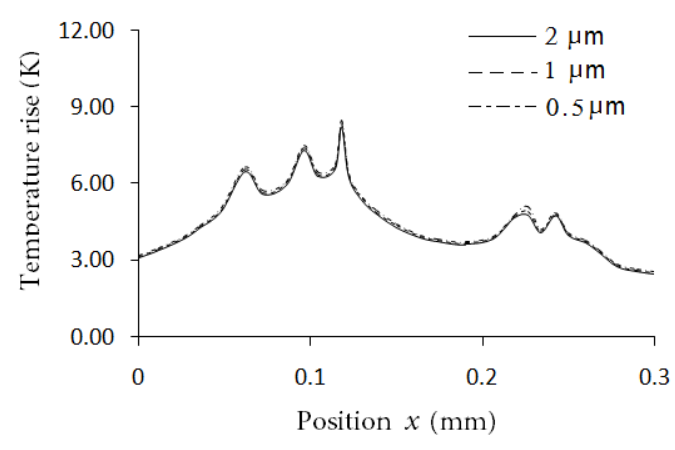

(b)

Fig. 7 Frictional shear stress and temperature rise distributions along the surface of the flat specimen in the tenth cycle from the elastic-plastic rough contact models with different element sizes in the contact zone: (a) frictional shear stress distributions; (b) temperature rise distributions.

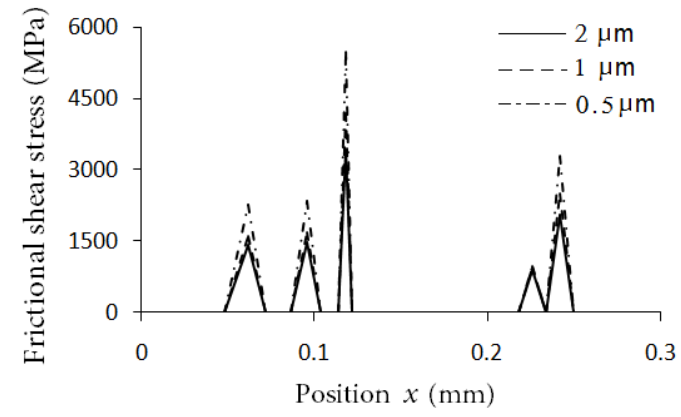

(a)

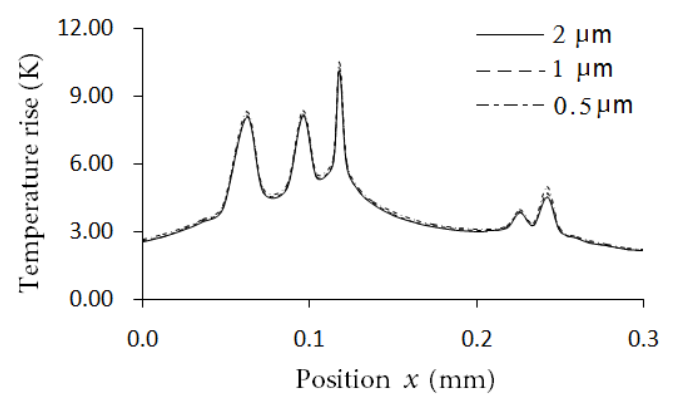

(b)

Fig. 8 Frictional shear stress and temperature rise distributions along the surface of the flat specimen in the tenth cycle from the elastic rough contact models with different element sizes in the contact zone: (a) frictional shear stress distributions; (b) temperature rise distributions.

\subsection{Frictional shear stress and temperature rise distributions}

The temperature rise distributions near the contact area after the first ten cycles (the temperature rise history result at different locations in Fig. 13 shows that the rise rate reaches a steady state after ten cycles) from the smooth contact and rough contact models are shown in Fig. 9 (a) and Fig. 9 (b) respectively. It can be seen that the temperature rise distribution from the rough contact model is very different to that from the smooth contact model. The highest temperature rise from the rough contact model is higher than that from the smooth contact model and there are several local temperature rise peaks in the rough contact zone. Since the contact stress and temperature rise distributions vary with 
the time during the fretting process, the comparison of frictional shear stress and temperature rise distributions along the surface of the flat specimen in the contact area at different time in the tenth cycle (when $t=0.0474 \mathrm{~s}, 0.0481 \mathrm{~s}$ and $0.0498 \mathrm{~s}$, the frictional shear stress and temperature rise at the positions of $x=0.06 \mathrm{~mm}, 0.12 \mathrm{~mm}$ and $0.22 \mathrm{~mm}$ along the surface of the flat specimen from the rough contact model get to the highest values respectively, and $t=0.0500 \mathrm{~s}$ is the end of the tenth cycle) between the smooth contact and rough contact are shown in Fig. 10 and Fig. 11 respectively.

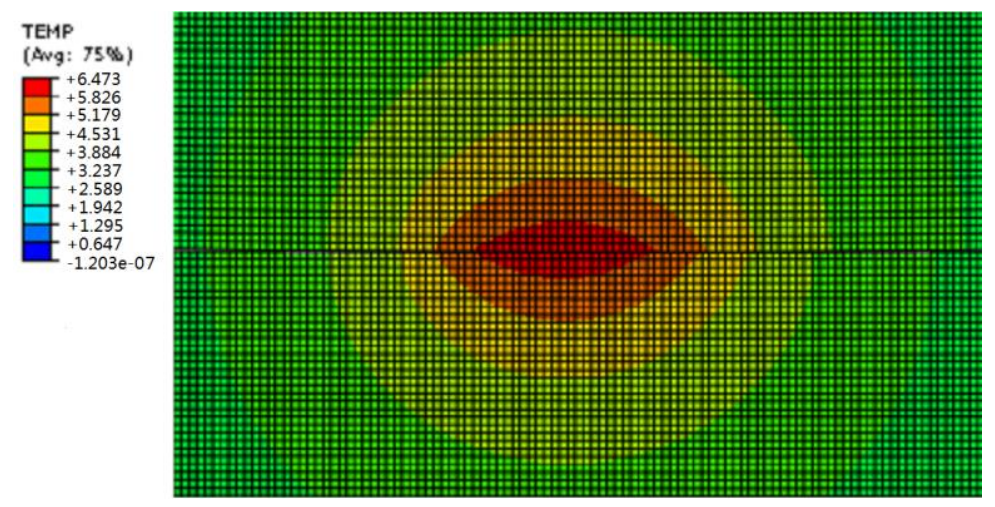

(a)

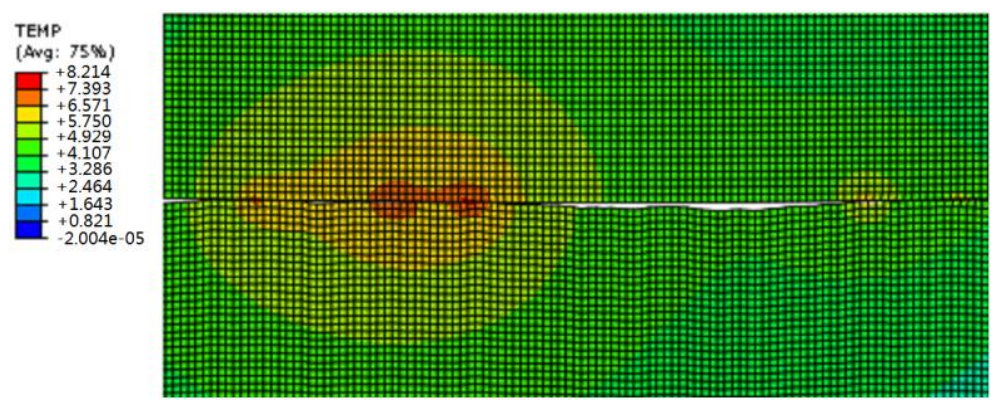

(b)

Fig. 9 Temperature rise distribution in the contact zone: (a) smooth contact; (b) rough contact.

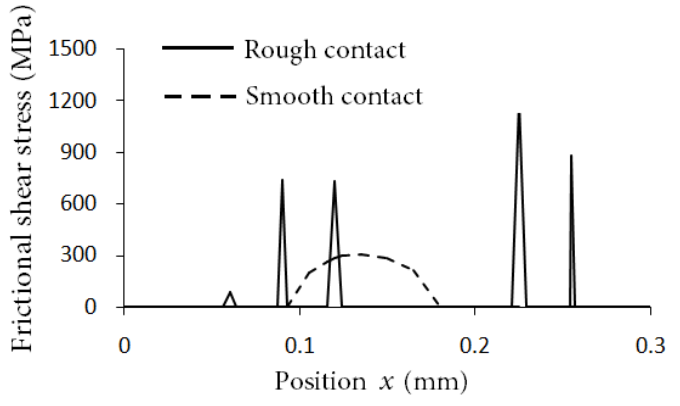

(a)

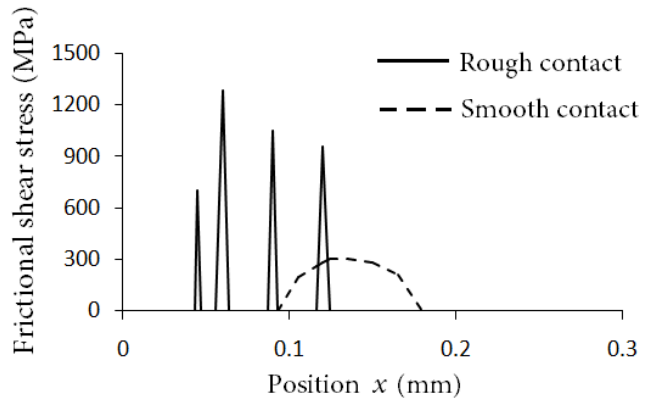

(b) 


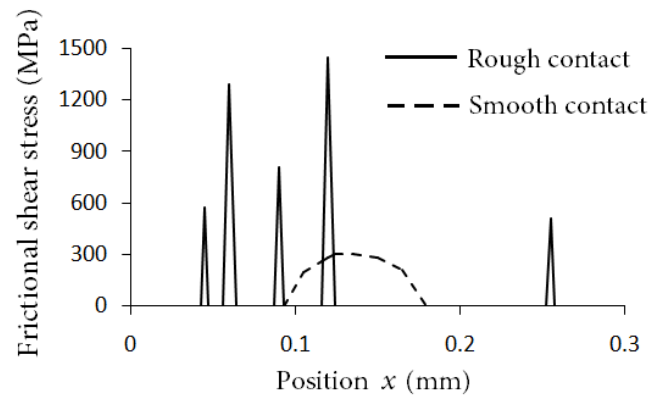

(c)

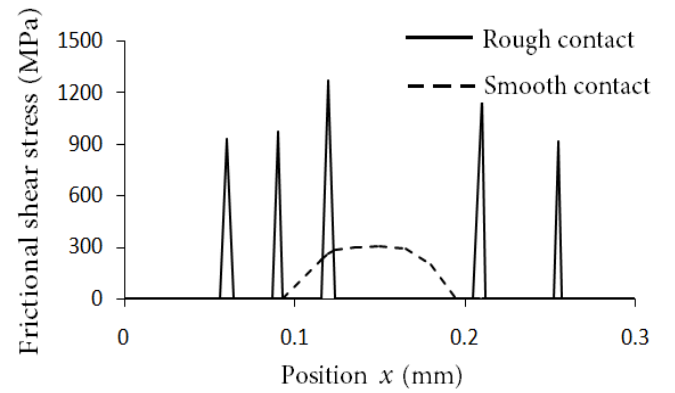

(d)

Fig. 10 Frictional shear stress distributions along the surface of the flat specimen in the tenth cycle from the smooth contact and rough contact models at: (a) $t=0.0474 \mathrm{~s}$, (b) $t=0.0481 \mathrm{~s}$, (c) $t=0.0498 \mathrm{~s}$, (d) $t=0.0500 \mathrm{~s}$.

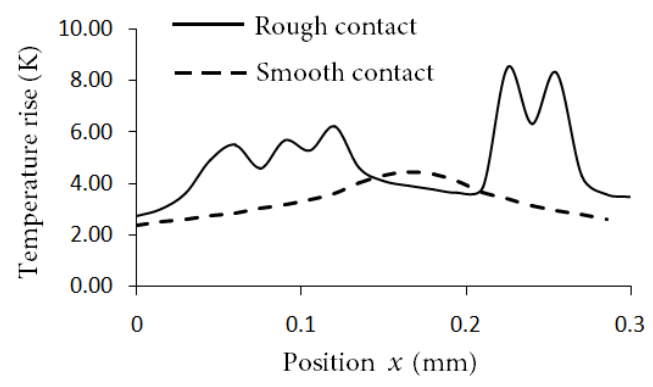

(a)

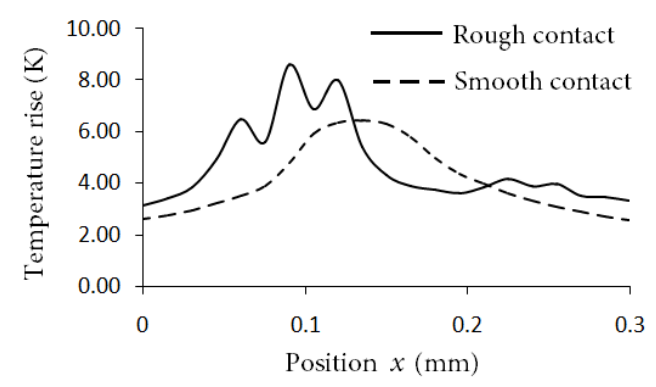

(c)

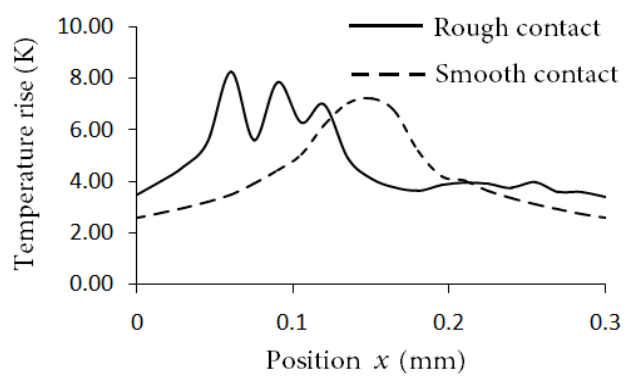

(b)

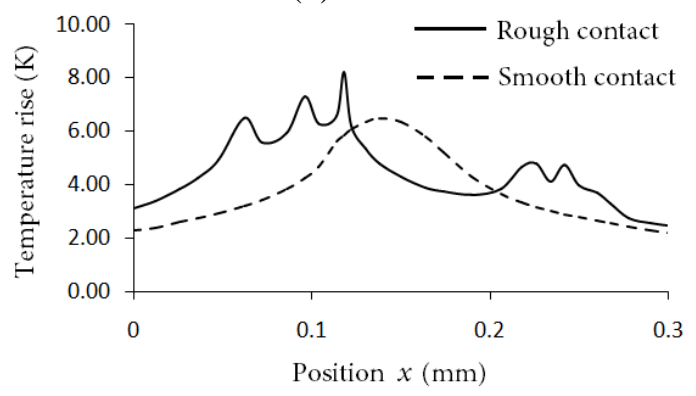

(d)

Fig. 11 Temperature rise distributions along the surface of the flat specimen in the tenth cycle from the smooth contact and rough contact models at: (a) $t=0.0474 \mathrm{~s}$, (b) $t=0.0481 \mathrm{~s}$, (c) $t=0.0498 \mathrm{~s}$, (d) $t=0.0500 \mathrm{~s}$.

From Fig. 10, it can be seen that the frictional shear stress distribution resulting from the rough contact model is discrete and the local shear stresses are highly concentrated compared with those from the smooth contact model. Moreover, the number and values of the frictional shear stress peaks from the rough contact model also change with the time during the fretting cycle. These result in substantially different temperature rise distributions in the rough contact model and the smooth contact models; in addition, values of the peak temperature rise predicted from the rough contact model also vary significantly with time as well (as shown in Fig. 11). Since the real contact area in the rough contact model is smaller than the contact area in the smooth contact model, the highest frictional shear stress and temperature rise from the rough contact model are much larger than those 
from the smooth contact model.

Fig. 12 shows the distribution of temperature rise in the subsurface of the flat specimen at locations with the highest temperature rise ( $x=0.12 \mathrm{~mm}$ in the rough contact and $x=0.15 \mathrm{~mm}$ in the smooth contact) after the first ten cycles. The figure shows that the gradients of temperature rise from both the smooth contact model and the rough contact model are very steep within a shallow subsurface (depth $=0 \sim 0.2 \mathrm{~mm}$ ) and this zone may be the 'thermally disturbed zone' described in Ref. 14 . The figure also shows that the temperature rise is very low $(\leq 0.3 \mathrm{~K})$ at a depth greater that $1.0 \mathrm{~mm}$. This phenomenon indicates that the significant temperature rise resulting from a fretting contact is limited to a very small zone surrounding the contact areas.

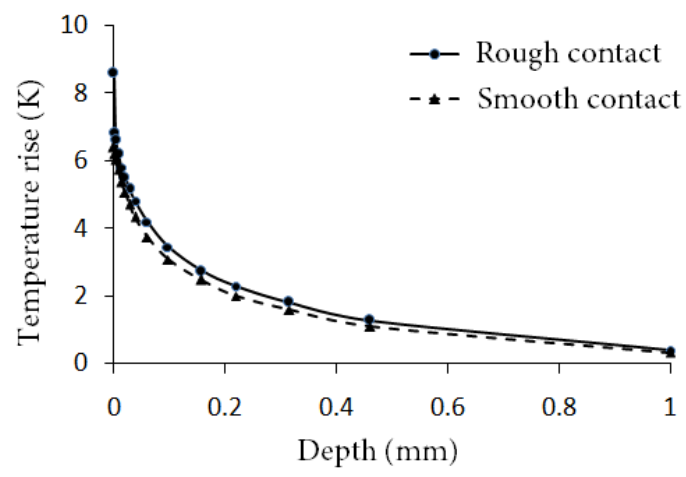

Fig. 12 Temperature rise distribution in the subsurface of the flat specimen at locations with highest temperature rise $(x=0.12 \mathrm{~mm}$ in the rough contact and $x=0.15 \mathrm{~mm}$ in the smooth contact $)$ after the first ten cycles.

\subsection{Time history of temperature rise}

Fig. 13 gives the time histories of temperature rise at different locations on the surface of the flat specimen including the middle of the contact area $(x=0.15 \mathrm{~mm})$ and the several positions at which the temperature gets to the local peak $(x=0.06 \mathrm{~mm}, 0.12 \mathrm{~mm}$ and $0.22 \mathrm{~mm}$ ) from the rough contact model and the time histories of temperature rise at the same locations from the smooth contact model in the first ten cycles. It can be seen that the temperature rises at different locations increase with the oscillatory behaviour, and the rise rate is fast at first and then falls as the situation reaches a steady state. The amplitude of the temperature rise in the middle of the contact area from the rough contact model is much lower than the amplitude of temperature rise at other locations because no contact exists at the middle of the contact area in this case; in contrast, the largest temperature rise amplitude in the smooth contact model occurs in the middle of the contact area. The differences in the amplitude of temperature rise at different locations in the rough contact model are relatively small (less than $1 \mathrm{~K}$ ), but those from the smooth contact model are much greater, and the amplitude of temperature rise in the smooth contact model decreases as the distance between the location and the middle of the contact area increases. 


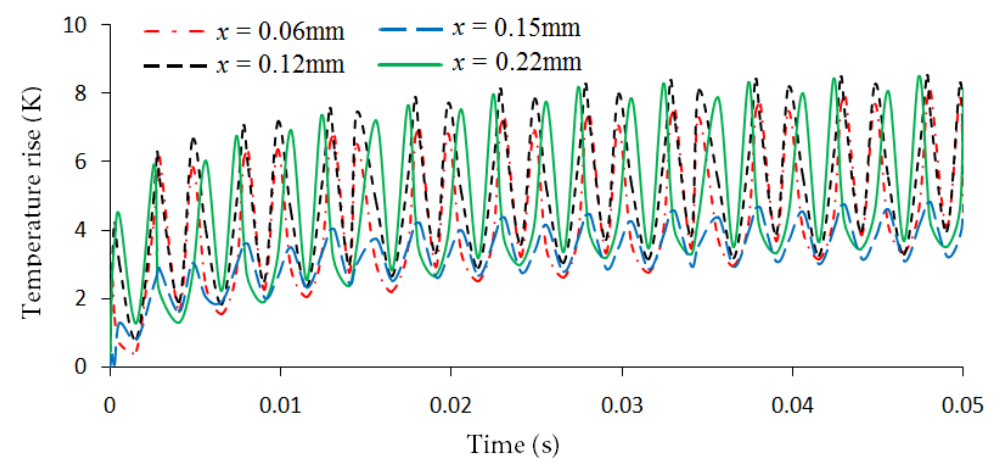

(a)

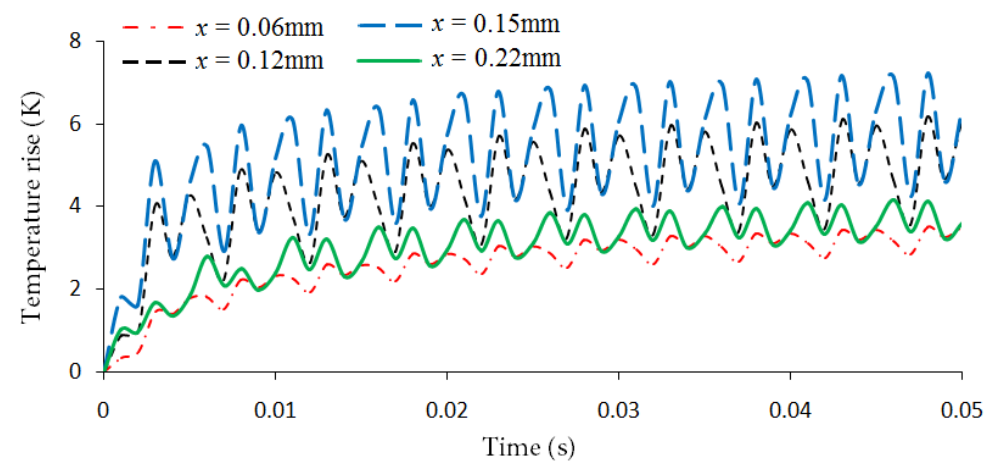

(b)

Fig. 13 Time histories of temperature rise at different locations of the flat specimen in the first ten cycles from: (a) rough contact model; (b) smooth contact model.

\subsection{Effect of plasticity, load and frequency}

In order to show the effect of plasticity, the rough contact model with elastic material (without plasticity) was built and the corresponding coupled temperature-displacement simulation was implemented. The effect of the inclusion of plasticity in the FE model is shown in Figure 14 where the contact pressure, frictional shear stress and temperature rise distributions along the surface of the flat specimen after the first ten cycles resulting from the rough contact model with and without plasticity are compared. Since the real contact area of rough surfaces is much smaller than that of smooth surfaces, plastic deformation occurs at some of the asperities. On account of the resulting surface flattening, the largest contact pressure is much lower than that from the elastic material model which results in a reduction in the largest frictional shear stress and thus a smaller value of the highest temperature rise (a reduction of $\sim 23 \%$ ).

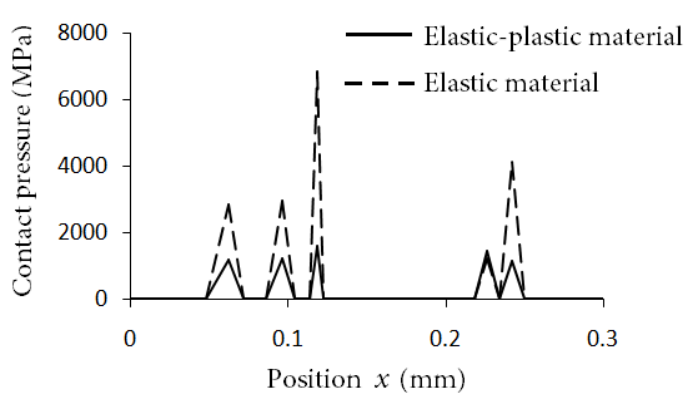

(a)

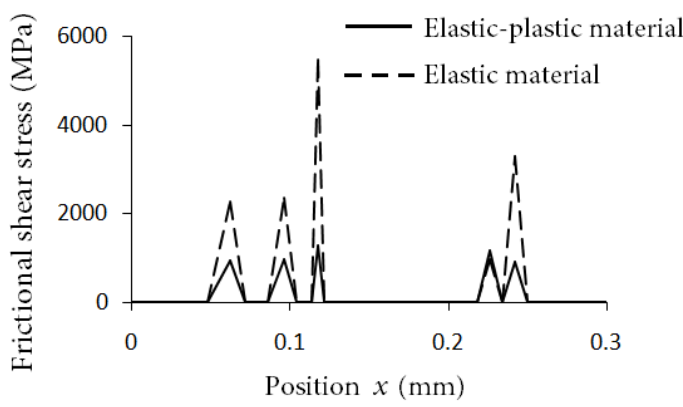

(b) 


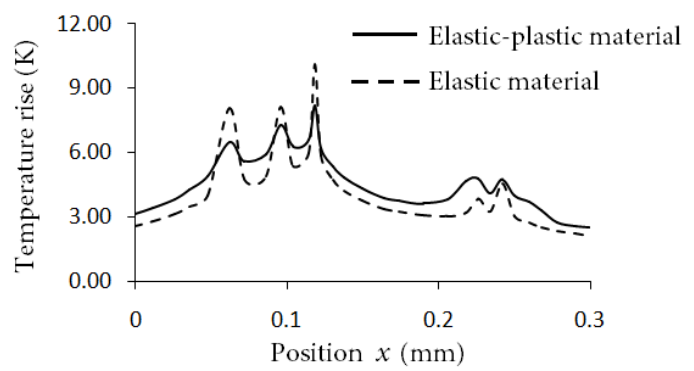

(c)

Fig. 14 Comparison of contact pressure, frictional shear stress and temperature rise distributions along the surface of the flat specimen after the first ten cycles from the elastic and elastic-plastic material models.

The effect of load was investigated by both increasing and decreasing the load by $100 \mathrm{~N}$ from its initial value of $250 \mathrm{~N}$. The simulated frictional shear stress and temperature rise distributions along the surface of the flat specimen after the first ten cycles under the loads of $150 \mathrm{~N}, 250 \mathrm{~N}$ and $350 \mathrm{~N}$ from the rough contact model are shown in Fig. 15. It can be seen that the temperature on the contact surface rises as the load increases. The increase in peak frictional shear stress with increasing applied load is not very significant (especially as the load is increased from $250 \mathrm{~N}$ to $350 \mathrm{~N}$ ) since the actual contact pressure does not increase very rapidly due to plastic deformation.

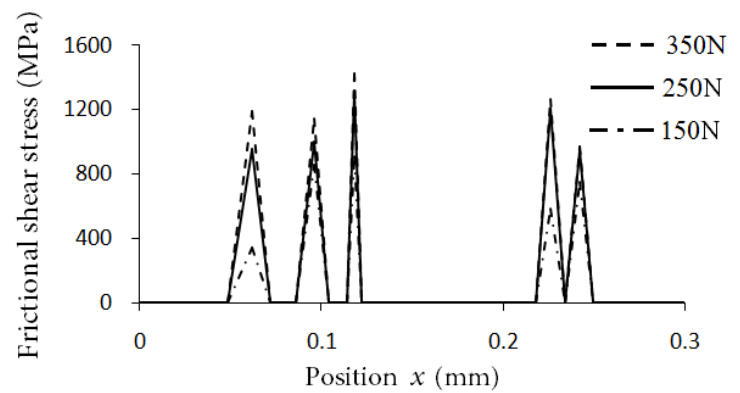

(a)

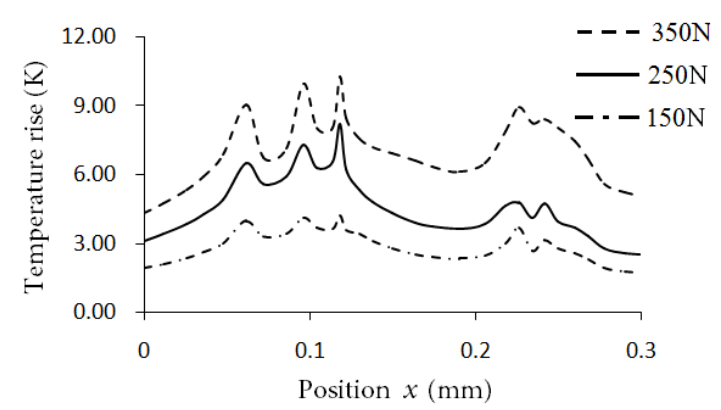

(b)

Fig. 15 Frictional shear stress and temperature rise distributions along the surface of the flat specimen after the first ten cycles under the various loads.

The effect of fretting frequency is investigated by changing the frequency to $100 \mathrm{~Hz}$ and $300 \mathrm{~Hz}$ (from its initial value of $200 \mathrm{~Hz}$ ). The simulated temperature rise distributions along the surface of the flat specimen after the first ten cycles from the rough contact model are shown in Fig. 16. It can be seen that the temperature along the surface of the flat specimen also increases with increasing frequency, although the variation in frequency does not result in changes to the frictional shear stress distribution. This is caused by the increase of the frictional heat flux density as the slip rate in Eq. (6) increases due to the increasing of frequency. 


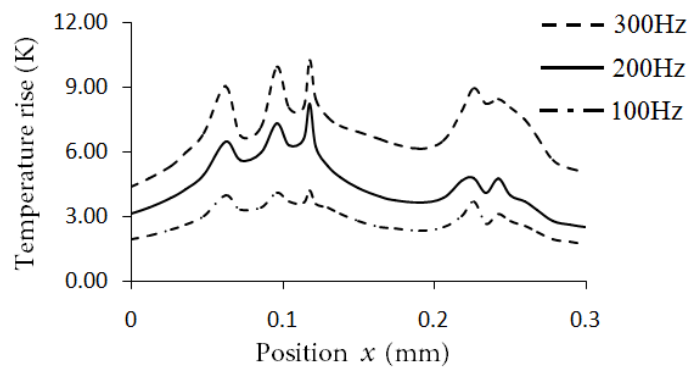

Fig. 16 Temperature rise distributions along the surface of the flat specimen after the first ten cycles under the various frequencies.

\section{Conclusions}

Roughness varies significantly depending on the scale and position along the length of a specimen. To implement a flexible and scale-independent rough profile in the FE model, the W-M function was used to characterize the surface profile as a fractal geometry in this study. The dynamic local frictional shear stress and temperature rise distributions for the rough and smooth cylinder-on-flat fretting contacts were simulated by the coupled temperature-displacement FE method.

Comparison of simulation results from smooth contact and rough contact models shows that the frictional shear stress distribution resulting from the rough contact model is discrete, time-varied and the local stresses are highly concentrated compared with that from the smooth contact model. These characteristics result in differences in the temperature rise distribution from the rough contact model when compared with that from the smooth contact model. The highest temperature rise from the rough contact model does not occur at the centre of the contact zone and its value is greater than that from the smooth contact model.

The gradients of temperature rise from both the smooth contact model and the rough contact model are very steep and non-linear within the shallow subsurface (depth $=0 \sim 0.2 \mathrm{~mm}$ ), and the temperature rise is very low $(\leq 0.3 \mathrm{~K})$ at a depth greater than $1.0 \mathrm{~mm}$. This indicates that the significant temperature rise resulting from a fretting contact under these conditions is limited to a very small zone surrounding the contact areas.

The temperature rise is observed to increase with the oscillatory behaviour and the rise rate is fast first and then slow to reach a steady state. The differences in the amplitude of temperature rise at different locations from the rough contact model are relatively small, but those from the smooth contact model are much greater; the smooth contact model indicates that the amplitude of the temperature rise decreases with increasing distance between the location and the middle of the contact area .

On account of material plasticity, the greatest contact pressure is much lower than those from elastic material model which results in much lower values of the maximum frictional shear stress and smaller values of maximum temperature rise.

The simulation results show that the temperature on the contact surface increases significantly when the load and frequency increase.

\section{Acknowledgements}

The authors would like to thank the Mitaka Kohki Co. Ltd. and Shared Labs Europe Ltd. for providing equipment and assistance in the measurement of surface roughness of the specimens. 
Thanks are also due to the graduate student Yixin Zhang at Beijing Institute of Technology for the assistance regarding description of surface roughness by fractal geometry.

\section{References}

[1] Attia H. Thermal constriction phenomenon in fretting: theory and implications. Tribol Int 2011, 44: $1352-1363$.

[2] Jin X, Sun W, Shipway PH. The role of geometry changes and oxide debris layers associated with wear on the local temperature field in fretting contacts. Tribol Int 2016; 102: 392-406.

[3] Pearson S, Shipway PH, Abere JO, Hewitt RAA. The effect of temperature on wear and friction of a high strength steel in fretting. Wear 2013; 303: 622-31.

[4] Colombie C, Berthier Y, Floquet A, Vincent L, Godet M. Fretting: load carrying capacity of wear debris. ASME J Tribol Trans 1984; 106:194-201.

[5] Rybiak R, Fouvry S, Bonnet B. Fretting wear of stainless steels under variable temperature conditions: introduction of a 'composite' wear law. Wear 2010; 268: 413-423.

[6] Fouvry S, Duó P, Perruchaut Ph. A quantitative approach of Ti-6Al-4V fretting damage: friction, wear and crack nucleation. Wear 2004, 257: 916-929.

[7] Jin X, Sun W, Shipway PH. Derivation of a wear scar geometry-independent coefficient of friction from fretting loops exhibiting non-Coulomb frictional behaviour. Tribol Int 2016, 102: 561-568.

[8] Lee H, Mall S. Some observations on frictional force during fretting fatigue. Tribol Lett 2004, 17: 491-499.

[9] Wang RH, Jain VK, Mall S. A non-uniform friction distribution model for partial slip fretting contact. Wear 2007; 262: 607-616.

[10] Kalin M. Influence of flash temperatures on the tribological behaviour in low-speed sliding: a review. Mat Sci Eng A 2004; 374: 390-397.

[11] Kalin M, Vižintin J. Comparison of different theoretical models for flash temperature calculation under fretting conditions. Tribol Int 2001; 34: 831-839.

[12] Ashby MF, Abulawi J, Kong HS. Temperature maps for frictional heating in dry sliding. Tribol Trans 1991; 34: 577-587.

[13] Greenwood JA, Alliston-Greiner AF. Surface temperatures in a fretting contact, Wear 1992; 155 : 269-275.

[14] Attia MH, Camacho F. Temperature field in the vicinity of a contact asperity during fretting. ASME-Publications-PED 1993, 67: 51-61.

[15] Wen J, Khonsari MM. Transient temperature involving oscillatory heat source with application in fretting contact. J Tribol 2007; 129: 517-527.

[16] Jin X, Shipway PH, Sun W. The role of frictional power dissipation (as a function of frequency) and test temperature on contact temperature and the subsequent wear behaviour in a stainless steel contact in fretting. Wear 2015; 330-331: 103-111.

[17] Lu W, Zhang P, Liu X, Zhai W, Zhou M, Luo J, Zeng W, Jiang X. Influence of surface topography on torsional fretting wear under flat-on-flat contact. Tribiol Int 2017; 109: 367-372.

[18] Kubiak KJ, Liskiewicz TW, Mathia TG. Surface morphology in engineering applications: Influence of roughness on sliding and wear in dry fretting. Tribol Int 2011; 44: 1427-1432. 
[19] Kubiak KJ, Mathia TG, Fouvry S. Interface roughness effect on friction map under fretting contact conditions. Tribol Int 2010; 43: 1500-1507.

[20] Svahn F, Kassman-Rudolphi Å, Wallén E. The influence of surface roughness on friction and wear of machine element coatings. Wear 2003; 254: 1092-1098.

[21] Sedlaček M. Podgornik B, Vižintin J. Influence of surface preparation on roughness parameters, friction and wear. Wear 2009; 266: 482-487.

[22] Reichert S, Lorentz B, Albers A. Influence of flattening of rough surface profiles on the friction behaviour of mixed lubricated contacts. Tribo Int 2016; 93: 614-619.

[23] Tayebi N, Polycarpou AA. Modeling the effect of skewness and kurtosis on the static friction coefficient of rough surfaces. Tribol Int 2004; 37: 491-505.

[24] Horng JH, Wei CC, Tsai HJ, Shiu BC. A study of surface friction and particle friction between rough surfaces. Wear 2009; 267: 1257-1263.

[25] Attia MH, Yovanovich MM. A model for predicting the thermal constriction resistance infretting. Proc ASME Symp Contact Probl Surf Interact Manuf Tribol Syst 1993; 67: 63-74.

[26] Attia MH, D'Silva NS. Effect of mode of motion and process parameters on the prediction of temperature rise in fretting. Wear 1985;106:203-24.

[27] Sayles RS, Thomas TR. Surface topography as a non-stationary random process. Nature 1978; 271:431-434.

[28] Majumdar A, Bhushan B. Role of fractal geometry in roughness characterization and contact mechanics of surfaces. ASME J Tribol 1990; 112: 205-216.

[29] Majumdar A, Bhushan B. Fractal model of elastic plastic contact between rough surfaces. ASME J Tribol 1991; 113:1-11.

[30] Mandelbrot BB. The fractal geometry of nature. New York: WH Freeman; 1983.

[31] Yin X, Komvopoulos K. An adhesive wear model of fractal surfaces in normal contact. Int J Solids Struct 2010; 47: 912-921.

[32] Komvopoulos K, Yan W. A fractal analysis of stiction in microelectromechanical systems. ASME J Tribol 1997; 119: 391-400.

[33] Zhang X, Xu Y, Jackson RL. An analysis of generated fractal and measured rough surfaces in regards to their multi-scale structure and fractal dimension. Tribol Int 2017; 105: 94-101.

[34] Ge S, Zhu H. Fractal of tribology. Beijing: China Machine Press; 2005.

[35] Hyde T R, Leen S B, McColl I R. A methodology for modelling the effects of nitriding on fatigue life. Proc IMechE, Part L: J Materials: Design and Applications 2008; 222: 1-14.

[36] Bennett C. Finite element modelling of the inertia friction welding of a CrMoV alloy steel including the effects of solid-state phase transformations. J Manuf Process 2015; 18: 84-91.

[37] Abaques analysis user's guide, version 6.4. Dassault Systèmes; 2014.

[38] Aghdam AB, Khonsari MM. Prediction of wear in reciprocating dry sliding via dissipated energy and temperature rise. Tribol Lett 2013; 50: 365-378.

[39] Warmuth AR, Shipway PH, Sun W, Fretting wear mapping: the influence of contact geometry and frequency on debris formation and ejection for a steel-on-steel pair. Proc. R. Soc. A 2015; 471: 20140291. 